\title{
Condiciones de accesibilidad y movilidad en el polígono industrial del río Ripoll (Sabadell) Un análisis de metodología múltiple ${ }^{1}$
}

\author{
Carme Miralles-Guasch \\ Montserrat Martínez Melo \\ Oriol Marquet Sardà \\ Universitat Autònoma de Barcelona. Departament de Geografia \\ carme.miralles@uab.cat \\ montserrat.martinez.melo@uab.cat \\ oriol.marquet@uab.cat
}

Recepción: enero de 2012

Aceptación: febrero de 2012

\section{Resumen}

El artículo analiza las condiciones de movilidad y de accesibilidad del PAE del río Ripoll de Sabadell. La metodología utilizada ha sido multiestratégica, puesto que se han combinado métodos cuantitativos, cualitativos y de observación directa. Además del contexto territorial y económico de la zona, la movilidad se determina por las características de los desplazamientos y por las percepciones de los trabajadores y las empresas. También se valoran los niveles de accesibilidad según el transporte público, el aparcamiento, la circulación de los vehículos pesados y el acceso en bicicleta y andando.

Palabras clave: movilidad; accesibilidad; industria; Sabadell.

Resum. Condicions d'accessibilitat i de mobilitat al polígon industrial del riu Ripoll (Sabadell): Una análisi de metodologia múltiple

L'article analitza les condicions de mobilitat i d'accessibilitat del PAE del riu Ripoll de Sabadell. La metodologia utilitzada ha estat multiestratègica, atès que s'hi han combinat mètodes quantitatius, qualitatius i d'observació directa. A més del context territorial i econòmic de la zona, la mobilitat s'hi determina per les característiques dels desplaçaments i per les percepcions dels treballadors i de les empreses. També s'hi valoren els nivells d'accessibilitat segons el transport públic, l'aparcament, la circulació dels vehicles pesants i l'accés amb bicicleta i caminant.

Paraules clau: mobilitat; accessibilitat; indústria; Sabadell.

1. Los autores quieren agradecer las aportaciones realizadas por el Ayuntamiento de Sabadell. Este artículo ha sido financiado por el proyecto CSO2010-18022 (subprograma GEOG. La perspectiva territorial, social y medioambiental en las investigaciones sobre movilidad y transporte. Un análisis desde la geografia). 
Résumé. Conditions d'accès et mobilité dans le polygone industriel du la rivière Ripoll (Sabadell): Analyse methodologie de multiples

L'article analyse les conditions de mobilité et d'accessibilité du PAE de la rivière Ripoll de Sabadell. Une méthodologie multi-stratégique a été utilisée, en combinant des méthodes quantitatives, qualitatives et d'observation directe. Outre le contexte territorial et économique de la zone, la mobilité est déterminée par les caractéristiques des déplacements et par les perceptions des travailleurs et des entreprises. Les niveaux d'accessibilité selon les transports publics, le parc de stationnement, la circulation des véhicules lourds, et l'accès en vélo et à pied sont aussi tenus en compte.

Mots clé: mobilité; accessibilité; industrie; Sabadell.

Abstract. Accessibility and Mobility in the Ripoll River Industrial Park of Sabadell: A MultiMethod Analysis

This paper analyzes mobility conditions in the Ripoll River industrial park located in the municipality of Sabadell (Spain). A multi-strategic methodological approach combining qualitative and quantitative methods along with direct observation is used. In addition to the territorial and economic context of the area, mobility is also affected by the characteristics of the displacements and the perceptions of both the workers and the enterprises located in the park. Different levels of accessibility such as public transport, parking, heavy vehicles and bicycle or pedestrian access are also taken into account.

Keywords: mobility; accessibility; industry; Sabadell.

\section{Sumario}

\section{Introducción 4. Análisis de la movilidad de}

2. El polígono de actividad económica los trabajadores del polígono del Ripoll del río Ripoll en su contexto 5. Valoración de la accesibilidad al PAE territorial y económico del río Ripoll

3. Metodología múltiple 6. Conclusiones

Bibliografía

\section{Introducción}

El artículo analiza las condiciones de movilidad y de accesibilidad de los trabajadores del polígono de actividad del río Ripoll, en Sabadell (Barcelona), situado en el cauce del río a su paso por la ciudad. Además del contexto territorial y económico de la zona, la movilidad se determina por las características de las infraestructuras, por la oferta de transportes públicos y por las percepciones que tienen sobre sus propios desplazamientos los trabajadores y las empresas. La aparición y la ubicación de los polígonos industriales tienen una lógica territorial sustentada en el urbanismo racionalista basado en la separación y la exclusión de las actividades urbanas y sus usos económicos (López, 1993). A la vez, persisten, de la primera etapa de la industrialización, criterios de 
aislamiento de la actividad industrial de las zonas residenciales de los núcleos urbanos, por las molestias que la actividad industrial provoca a la población (Esteban, 2008). Estos dos criterios han sido la base conceptual de la localización aislada y suburbana de los polígonos industriales en las ciudades del mundo occidental en la segunda mitad del siglo xx.

En Cataluña, además, la ubicación de las actividades industriales tiene dos dinámicas propias, superpuestas a las ya mencionadas. La primera obedece a la lógica del siglo XIX y principios del Xx, cuando las industrias, por cuestiones energéticas, se ubicaban a lo largo de los ríos. La segunda tiene su origen en la política industrial del último tercio del siglo pasado, cuando la lógica política fomentaba un área industrial aislada en cada municipio. Ello ha provocado que, en Cataluña, hubiera, en 2006, 1.748 polígonos de actividad económica, que ocupaban unas 32.240 hectáreas, un 18\% del suelo urbano o urbanizable (Miralles-Guasch y Donat, 2008). Polígonos a menudo subutilizados, de dimensiones no apropiadas y con deficiencias, especialmente respecto a la accesibilidad y a las condiciones de movilidad de sus trabajadores (Cebollada, 2008).

Sin embargo, en los últimos años, la aproximación teórica al planeamiento territorial y al propio concepto de polígono industrial ha ido cambiando. En primer lugar, en estos emplazamientos, se van ubicando actividades terciarias. De ello deriva el cambio de denominación — «polígonos de actividad económica» (PAEs)—, que amplía su registro funcional. También, y con el objetivo de crear áreas urbanas mixtas donde convivan actividades económicas y residenciales, en la línea de una reinvención de la ciudad mediterránea densa, variada y opuesta a los principios del urbanismo funcionalista, se están conceptualizando nuevas ideas urbanas que no aíslen sus funciones (Esteban, 2008), aunque pueden persistir algunas incompatibilidades relacionadas con los tamaños formales de las empresas y con algunas de sus actividades. En este sentido, el planeamiento urbanístico de Cataluña entre 2004 y 2010 quiso ahondar en dicho nuevo paradigma urbano y subsanar déficits, tanto en términos urbanísticos como de accesibilidad (PTOP, 2010).

La hipótesis de partida de este análisis es que los condicionantes de la movilidad y la accesibilidad a los polígonos industriales no pueden cambiar sólo desde políticas de transporte. A éstas se le tienen que añadir políticas de planificación territorial que, como el planeamiento propuesto entre 2004 y 2010 en Cataluña, ahonden en no aislar funciones propias de las ciudades. En este sentido, los PAEs tendrían que ser espacios multifuncionales, con la inclusión de residencia y equipamientos urbanos.

\section{El polígono de actividad económica del río Ripoll en su contexto territorial y económico}

El PAE del Ripoll es una zona industrial ubicada en el cauce del río Ripoll a su paso por el municipio de Sabadell. Se trata de un espacio industrial tradicional de la ciudad, con pendientes pronunciadas que dificultan el acceso, con una forma lineal, de trazado discontinuo y desconectado en sus distintas partes 
(Barbé, 1971; Benaul et al., 1994; Deu et al., 2000) y con una conectividad interna inexistente, que requiere de las redes viarias de la ciudad para transitar a lo largo del polígono. El Ripoll no es un polígono estándar, lo que supone ciertas potencialidades, pero también demanda de una gestión específica de aquellos componentes que limitan su funcionamiento y su competitividad, como son la accesibilidad y las condiciones de movilidad de sus trabajadores (Pla Mobilitat Urbana de Sabadell, 2007).

La proximidad a la ciudad, aunque ubicado en su parte más externa, es uno de sus mayores potenciales en relación con el mercado de trabajo y con los servicios urbanos. También la cercanía a otros entornos industriales y a la red viaria, además de su paisaje, son los factores más valorados. Las características que le otorgan más dificultad son los accesos y la limitada conectividad interna del polígono, por lo que se establecen tres subzonas dentro de éste (norte, centro y sur), bastante distintas entre ellas.

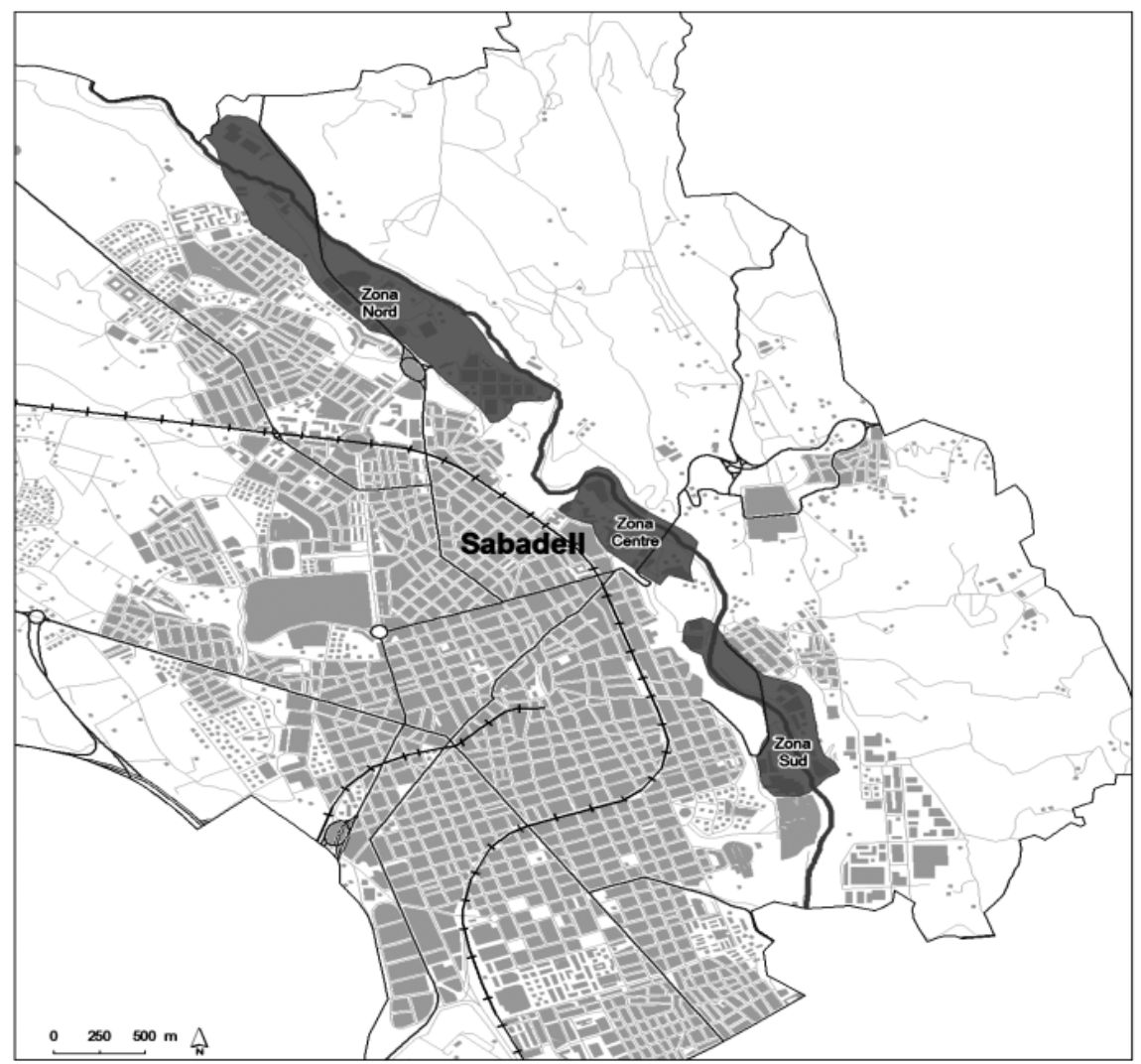

Mapa 1. Situación del polígono de actividad económica del río Ripoll (Sabadell). Fuente: elaboración propia. 
En los últimos años, la actividad industrial en el polígono ha ido disminuyendo, solo en 2009 el censo de empresas pasó de 75 a 59, lo que representa un descenso del $28 \%$, de las que 11 han cerrado y 8 se han ubicado en otros entornos, aunque también han aparecido cinco empresas nuevas. El sector textil, como anteriormente en toda la ciudad de Sabadell, es aún el predominante, con un $40 \%$ de la actividad, seguido del metalúrgico (un 17\%).

El establecimiento del parque fluvial, a finales de la década de 1990, posicionó a la industria en un nuevo contexto, puesto que pasó a convivir con otras actividades emergentes relacionadas con su entorno natural y de ocio. La industria ha tenido que adecuarse a las normativas urbanísticas que protegen este medio, aunque también le otorga nuevas potencialidades, como las actividades relacionadas con el medio ambiente, a la vez que le ofrece un entorno natural y paisajístico único.

El PAE del Ripoll se percibe como bastante desordenado, con distintos paisajes abandonados, fruto de la falta de planificación de conjunto y sin una identificación unitaria. Los esfuerzos de un planeamiento conjunto se han focalizado en la recuperación medioambiental del cauce del río y en la adecuación del espacio como lugar de ocio, en relación con sus huertos urbanos. A esto cabe añadir que este polígono se localiza en un entorno donde existen muy buenos espacios industriales y donde la oferta del suelo supera claramente la demanda (Cebollada y Miralles-Guasch, 2006). Esta situación en conjunto hace que el PAE del Ripoll tenga que afrontar retos importantes, con estrategias que orienten y canalicen su futuro.

En la actualidad, la población trabajadora del recinto es de unas 780 personas, de las cuales más de dos terceras partes son hombres, con una media de edad de 42 años, prácticamente la mitad ha cursado estudios secundarios y un $25 \%$ son universitarios. La mayor parte son asalariados, aunque un $8,3 \%$ son autónomos y empresarios. La inmensa mayoría de los asalariados tienen contratos indefinidos (un 93,2\%), por lo que la tasa de temporalidad es baja. El 63\% de la plantilla está formada por trabajadores dedicados a la producción $y$, entre ellos, predominan los obreros cualificados.

\section{Metodología múltiple}

La investigación se ha diseñado desde una perspectiva multiestratégica, mediante la combinación de técnicas cuantitativas y cualitativas. Las técnicas de análisis que se han utilizado han sido las siguientes:

1. Un análisis cuantitativo, en el que se han barajado datos primarios y secundarios. Entre las fuentes secundarias con las que se ha trabajado, se encuentran el censo de empresas del municipio de 2009 (Ajuntament de Sabadell, 2009), el padrón municipal de habitantes, el Catálogo de Actividades Económicas del Vallès Occidental y el mapa de las áreas industriales de Cataluña (UPIC, 2010). Para obtener los datos primarios, se realizaron encuestas a empresas y a trabajadores. En ambos casos, las encuestas fueron 
autoadministradas, enviadas por correo y recogidas personalmente en cada una de las empresas, cuyo universo era de 59. De ellas, 29 (un 44,1\%) respondieron el cuestionario. Los trabajadores eran unos 780 , de los que participaron 169 , un $21,7 \%$.

2. Para el análisis cualitativo, se realizaron entrevistas personales a agentes clave para el conocimiento y la comprensión del entorno: sindicatos, entidades medioambientales y organismos de intervención en el espacio del Ripoll. En total, se hicieron siete entrevistas de una hora de duración.

3. Con el objetivo de conocer las características de las infraestructuras de la zona, se ha realizado un exhaustivo trabajo de campo in situ para poder valorar ciertos aspectos. Concretamente, se han realizado ocho salidas de observación. Los elementos observables se han registrado de forma sistemática de acuerdo con una escala de 0-10.

\section{Análisis de la movilidad de los trabajadores del polígono del Ripoll}

\subsection{Las condiciones de los desplazamientos al polígono}

Las condiciones de los desplazamientos vienen marcadas por la oferta de transportes públicos o colectivos que permiten llegar a la zona y por las posibilidades de uso de los transportes privados por parte de las personas que trabajan en ella, además de las infraestructuras viarias que dan acceso al polígono.

Al tratarse de una zona integrada en la trama urbana consolidada del municipio, algunas líneas de autobuses urbanos recorren el perímetro del polígono, aunque con diferencias notables según los distintos sectores. El centro es donde menor cobertura hay, en cambio, el norte y el sur tienen servicios aceptables. Por el contrario, el transporte de empresa es inexistente.

El transporte privado es el gran protagonista de la movilidad en el polígono, y las características de sus trabajadores así lo confirman. El 96\% dispone de carné de conducir, una cifra muy superior a la de la población mayor de 18 años de Sabadell (un 69\%) e incluso superior a la de la población laboral (un 83\%). Además, el 92,3\% tiene a su disposición coche y/o motocicleta, una cifra muy superior a la de la población general, que es del 62\% (Pla Mobilitat Urbana de Sabadell, 2007). A ello se le tiene que sumar que el $70 \%$ de las empresas, tal y como dispone la normativa, tienen espacio propio para aparcar.

Al igual que la actividad industrial, las infraestructuras viarias están condicionadas por la orografía: en el norte, a través de la B-124 en conexión con el municipio de Castellar, en proceso de desdoblamiento; en el centro, a través de la C-1413, eje que atraviesa Sabadell y que se bifurca para enlazar con el municipio de Sentmenat (C-1413a) y Palau-Solità i Plegamans (C-155) y que, debido a sus precarias condiciones de circulación, está en fase de remodelación; en el sur, a través de la BV-2432, que conecta el centro de la ciudad con el barrio periférico de Torre-Romeu, que se sitúa en la otra orilla y que enlaza con otro polígono y con la ciudad de Mollet a través de la B-140. Se trata de la 
única vía que reúne las condiciones adecuadas para la circulación de vehículos y de personas, además de bicicletas.

Existe un carril bici que enlaza todos los sectores del río, sin aparcamientos para bicicletas y que no conecta el polígono con la ciudad, sino que es un carril aislado a lo largo del río, es decir, construido con la finalidad de recorrer la zona fluvial y no de unirlo a la ciudad.

\subsection{Las características de la movilidad de los trabajadores}

La movilidad de los trabajadores viene determinada por los niveles de congestión que se originan en la red viaria, que aquí se trata a nivel perceptivo, por el número de desplazamientos, por los medios de transporte utilizados y por los costes que representan.

Los agentes sociales entrevistados manifiestan que las tres vías de acceso al polígono, antes citadas, están congestionadas en hora punta, pero todos ellos también coinciden en afirmar que estos problemas no derivan del tráfico generado por él. La intensidad del tráfico que genera la zona se considera marginal respecto al que absorbe el conjunto del viario. Un viario que conecta la ciudad con un entorno (Castellar, Polinyà, etc.) donde existen extensas zonas de actividad económica, generadoras de grandes intensidades de tráfico, por lo que hay coincidencia en que no es desde la escala del polígono donde se tiene que abordar el análisis de dicho viario, sino desde una escala supramunicipal. A la vez, se percibe que la trama municipal de la ciudad, especialmente la regulación semafórica de la Gran Vía, condiciona el tráfico en los accesos y en el interior.

Según la encuesta realizada a los trabajadores, éstos hacen una media de 3,3 viajes en día laborable. Casi el 48\% solo hace dos desplazamientos al día y cerca del $45 \%$ realiza cuatro. El número de movimientos está relacionado con la tipología de la jornada: más del $90 \%$ de los que trabajan en jornada intensiva solo hacen dos viajes. En cambio, los que tienen jornada partida, más del $60 \%$, van a comer a casa, lo que significa cuatro viajes al día. La posibilidad de ir a casa a comer indica que la congestión no es muy intensa en las horas intermedias del día, ya que ésta no impide dichos desplazamientos a 6 de cada 10 trabajadores.

El 80,7\% de estos desplazamientos se realiza en vehículo privado, mayoritariamente en coche. Dentro del 5,3\% de los desplazamientos que se hacen con medios colectivos, destaca el 2,3 del autobús y el 3\% de desplazamientos en los que, además del autobús, los trabajadores tienen que andar para llegar al puesto de trabajo. Más de la mitad de los usuarios del bus añaden el andar como transporte diferenciado, claro indicador de la distancia de las paradas respecto al puesto de trabajo.

Los costes del transporte están claramente asociados a la distancia y, por tanto, al lugar de residencia, tanto en tiempo como en carburante utilizado. Así, los costes para los trabajadores que residen en Sabadell son aproximadamente la mitad que para el resto de trabajadores. Mientras que una persona que no vive en Sabadell tarda una media de 32 minutos para el conjunto de 
desplazamientos que realiza en una jornada y se gasta 115 euros en carburante como media mensual, un residente en Sabadell tarda 16 minutos y se gasta 55 euros en carburante.

\subsection{Las actitudes ante la movilidad}

Es interesante ver cómo las percepciones de los agentes sociales segregan claramente entre la movilidad ocupacional y la recreativa, que tiene otras pautas. Así, los que viajan en coche, mayoritariamente, tienen por motivo el trabajo. Los medios no motorizados o los públicos se asocian a las personas que utilizan el lecho del río para actividades recreativas o las que se dirigen a los huertos urbanos de la zona. Los datos de la encuesta de Movilidad Cotidiana de Cataluña de 2006 (ATM, 2006) confirman estas percepciones, pues, en términos generales, el uso del transporte privado para ir a trabajar es superior a los desplazamientos generados por otros motivos.

En términos cuantitativos, y según la encuesta realizada a los trabajadores del polígono, más del $50 \%$ de los que utilizan el medio privado declaran que lo hacen porque es más rápido. Sin embargo, el $77 \%$ de las respuestas están relacionadas con las deficiencias del transporte público: la falta de oferta adecuada, de regularidad o puntualidad o directamente incompatibilidad de horarios con el transporte público. También dentro del colectivo que no utiliza el coche, es significativo que un $44 \%$ declare que, en su caso, es más rápido y que el mismo porcentaje diga que no lo utiliza por no disponer de coche o de carné de conducir. A pesar de que la mayoría de conductores se declaran dispuestos a compartir el coche para llegar al trabajo, solo un 12,5\% lo comparte.

\section{Valoración de la accesibilidad al PAE del río Ripoll}

La valoración de la accesibilidad analiza la situación a partir de observaciones realizadas en el propio ámbito de estudio, además de las entrevistas a los agentes sociales y de las encuestas realizadas a empresarios y trabajadores. Tres fuentes de información para valorar las cuatro dimensiones más significativas en torno a la accesibilidad: el transporte público, el aparcamiento, la circulación de vehículos pesados y los accesos en transporte no mecanizado.

En el transporte público, la percepción de los agentes sociales es unánime, al considerar que solo el autobús es el medio de transporte apropiado para esta zona, aunque, según sus apreciaciones, el servicio que presta es insuficiente, por lo que sería conveniente incrementar su competitividad frente a los medios privados a través de carriles segregados o mediante la ampliación de las líneas existentes. Estas percepciones coinciden con las valoraciones que hacen los trabajadores y las empresas, mientras que los primeros lo puntúan en un 3,7 sobre 10, los segundos lo hacen en un 4 .

Sin embargo, estas percepciones se pueden matizar con la observación directa, pues ésta permite detallar la cobertura desigual del transporte público en el polígono. Mientras que la zona centro queda demasiado lejos de cualquier 
parada, la zona sur está mejor servida y la zona norte conforma un término medio. La puntuación en la observación dada en estas zonas es de 3,3, 6,3 y 10 , respectivamente.

A pesar de todo, uno de los mayores déficits no es la oferta de transporte público, sino la información, pues más de un tercio de los trabajadores declara no tener ningún conocimiento del servicio. Y entre los que sí dicen tenerlo, más de un $25 \%$ no sabe valorarlo, porque realmente desconoce el funcionamiento. Entre los que ofrecen una valoración, solo la puntualidad, la seguridad y la proximidad a la parada del polígono obtienen una evaluación por encima de cinco (sin sobrepasar el 5,3). La comodidad, la calidad global, el tiempo de trayecto, la frecuencia de paso y la combinación horaria se quedan por debajo de esta cifra.

Según la percepción de los agentes sociales entrevistados, no existe ningún problema de aparcamiento en el polígono. En él existe suficiente espacio para que los que acceden en transporte privado aparquen en espacios públicos o en recintos de las propias empresas. La observación realizada coincide con estas opiniones, con una valoración de 7,8 y, además, se observa una mínima indisciplina en el estacionamiento, lo que confirma una oferta adecuada a la demanda. Los trabajadores dan mayor puntuación a este aspecto, puesto que lo valoran con un 7, en cambio las empresas lo estiman en un 5,6, puntuación relacionada con la percepción del mal estado del aparcamiento específico para camiones.

Sin embargo, este aspecto de los vehículos pesados, que, por las características de la zona, tendría que estar en el centro del debate, no aparece como importante en las entrevistas realizadas. Esto puede ser debido a que es cada vez menor la circulación de esta tipología de vehículos, por la reducción de la actividad industrial del polígono y por la elevada pendiente de los accesos, que cuestionan la seguridad. Los trabajadores valoran más positivamente este aspecto $(5,5)$ que las propias empresas $(4,8)$. La observación realizada lo puntúa en un 6,6.

Al tratarse de un polígono pegado a la trama urbana de la ciudad, aunque con difíciles accesos en transportes no mecanizados (ir andando y en bicicleta), es interesante la valoración para acceder al polígono. La percepción generalizada de los entrevistados es que resulta difícil llegar en transportes no mecanizados, debido a los déficits en los accesos, principalmente por la insuficiencia de aceras y la inexistencia de carriles bici en relación con las empresas. Estas infraestructuras en el Ripoll están más diseñadas para el ocio, previo acceso en transporte privado o bus. También existen problemas de seguridad relacionados con la iluminación.

Sin embargo, los mayores déficits se encuentran en las infraestructuras para la circulación en bicicleta, ya que, mientras éstas tienen una valoración de la observación directa de 4,3, las aceras y los circuitos a pie alcanzan un 7,2. Mejor en las zonas norte y sur que en el centro. La valoración de las empresas es mucho menor, pues solo otorgan un 5,1 , y el $42,3 \%$ de ellas considera que no es seguro llegar caminando. Para los trabajadores, esta proporción aún es más elevada, puesto que llega al 50\%. La falta de aceras, la inseguridad y la intensidad del tráfico rodado son las razones que sostienen estas cifras. 


\section{Conclusiones}

Las condiciones de movilidad y accesibilidad del PAE del Ripoll en Sabadell se analizan desde una metodología múltiple, donde se relacionan los resultados de los análisis cuantitativos a empresas y trabajadores con las conclusiones de las entrevistas cualitativas, además de las valoraciones de las observaciones in situ. Estas tres perspectivas analíticas, junto con las fuentes secundarias de información disponibles, permiten valorar la movilidad y la accesibilidad del polígono, puesto que relacionan su oferta (red viaria y servicios de transporte público) y las condiciones objetivas de sus trabajadores en relación con el uso del transporte privado, con sus opciones personales de movilidad y con las percepciones que tienen sobre ella.

El PAE del río Ripoll es un polígono industrial tradicional en el que se están integrando actividades terciarias y lúdicas, pegado a la propia trama de la ciudad, aunque con desniveles pronunciados, con una oferta de transporte público adecuada en ciertas partes y con unos hábitos de movilidad por parte de sus trabajadores similares a los de los trabajadores de cualquier polígono ubicado fuera de la ciudad. Empresas y trabajadores organizan sus desplazamientos bajo la perspectiva del vehículo privado, por lo que otros tipos de circulación, sea ir a pie, en bicicleta o en transporte público, no se ubican en las posibilidades que barajan las actividades empresariales. En cambio, sí se utilizan si la actividad realizada en la zona está relacionada con las ofertas lúdicas que en ella se ofrecen.

Esta constatación reafirma la hipótesis inicial, pues el cambio modal en los desplazamientos por motivos de trabajo no puede tratarse solo desde la oferta de transportes alternativos al privado. El aislamiento de las actividades industriales y la percepción que los trabajadores tienen de éste hacen que los transportes públicos y no motorizados no estén incluidos en sus opciones personales, incluso en lugares industriales pegados a la trama urbana. Es por ello que los cambios de reparto modal, con el objetivo de reducir los niveles de utilización del transporte privado, tienen que promoverse, también, desde la multifuncionalidad urbana, que opta por los espacios donde industria, servicios y residencia estén mucho más próximos.

\section{Bibliografía}

Ajuntament de Sabadell (2009). Censo de empresas del municipio de Sabadell. Autoridad Transporte Metropolitano (ATM) (2006). Encuesta de Movilidad Cotidiana. Barcelona: ATM y Generalitat de Catalunya.

Barbé, L. (dir.) (1971). Dinámica y perspectiva del Vallès. 1969: La industrialización. Vol. 10. Sabadell: Caja de Ahorros de Sabadell.

Benaul, J.M.; Calvet, J. y Deu, E. (1994). Industria i Ciutat: Sabadell, 1800-1980. Barcelona: Fundació Bosch i Cardellach. Publicacions de l'Abadia de Montserrat.

Cebollada, A. (2008). "L'accessibilitat als polígons industrials: una assignatura pendent». Barcelona: Institut d'Estudis Regionals i Metropolitans de Barcelona. Papers, 45. 
Cebollada, A. y Miralles-Guasch, C. (2006). Diagnosi de l'accessibilitat als polígons industrials de Can Mitjans i Can Tries: Anàlisi de l'oferta i la demanda de transport. Bellaterra: Consell Comarcal del Vallès Occidental i Institut d'Estudis Regionals i Metropolitans de Barcelona.

Deu, E.; Calvet, J.; Marín, M. y Sala-Sanahuja, J. (2000). Sabadell al segle XX. Vic: Eumo Editorial.

Esteban, J. (2008). «Els espais d'activitats econòmiques en el planejament territorial». Barcelona: Institut d'Estudis Regionals i Metropolitans de Barcelona. Papers, 45.

Institut d’Estudis Regionals i Metropolitans de Barcelona (2007). Pla Mobilitat Urbana de Sabadell. Barcelona. [Material fotocopiat]

LÓPEZ DE LuCio, R. (1993). Ciudad y urbanismo a finales del siglo XX. Valencia: Servei de Publicacions de la Universitat de València.

Miralles-Guasch, C. y Donat, C. (2008). «Anàlisi de l'oferta i la demanda de polígons d'activitat a Catalunya». Barcelona: Institut d'Estudis Regionals i Metropolitans de Barcelona. Papers, 45.

PTOP (2010). Pla Territorial Metropolità de Barcelona. Volum I. Barcelona: Generalitat de Catalunya. Departament de Política Territorial i Obres Públiques.

Unió de Polígons Industrials de Catalunya (UPIC) (2010). Catálogo de Actividades Económicas del Vallès Occidental y el mapa de las áreas industriales de Catalunya [en línea]. <http://www.upic.es>. 